\title{
Doses de potássio e cálcio no crescimento da planta, na produção e na qualidade de frutas do morangueiro em cultivo sem solo
}

\author{
Potassium and calcium doses on plant growth, fruit yield and quality of strawberries in soilless \\ cultivation
}

\author{
Jerônimo Luiz Andriolo ${ }^{I *}$ Djeimi Isabel Jänisch ${ }^{\mathrm{I}}$ Odair José Schmitt ${ }^{\mathrm{I}}$ Miriane Dal Picio $^{\mathrm{I}}$ \\ Francieli Lima Cardoso ${ }^{\mathrm{I}}$ Lígia Erpen ${ }^{\mathrm{I}}$
}

RESUMO

Com o objetivo de avaliar o efeito de doses de potássio fornecido pela fertirrigação e de cálcio na parte aérea sobre o crescimento da planta, a produção e a qualidade de frutas do morangueiro em cultivo sem solo, plantas de morangueiro foram cultivadas com solução nutritiva contendo $9\left(T_{1}\right), 6\left(T_{2}\right.$, testemunha) e 4,28mmol $L^{-1}\left(T_{3}\right)$ de $K^{+}$e com a solução testemunha $\left(T_{2}\right)$ suplementada uma vez por semana por pulverizações foliares de cálcio nas concentrações de 2,5 $\left(T_{4}\right)$ e $5 \mathrm{~g} \mathrm{~L}^{-1}$ de $\mathrm{CaCl}_{2}\left(T_{5}\right)$. O crescimento da planta foi reduzido no tratamento com a concentração de potássio mais elevada $\left(T_{1}\right)$, e o maior IAF foi obtido na dose mais elevada de $\mathrm{CaCl}_{2}$ $\left(T_{5}\right)$. A maior produção de frutas foi obtida em $T_{2}$ e $T_{3}$, a acidez (AT) foi maior em $T_{1}$, enquanto o teor de sólidos solúveis (SST) e a relação SST/AT não diferiram significativamente entre os tratamentos. Concluiu-se que o aumento da concentração de potássio na solução nutritiva diminui o crescimento, a produção e a qualidade das frutas de morango e que a aplicação de cálcio na parte aérea das plantas por meio do $\mathrm{CaCl}_{2}$ reduz a produção de frutas.

Palavras-chave: Fragaria $x$ ananassa, substrato, solução nutritiva, cátions.

\section{ABSTRACT}

In order to evaluate the effect of potassium doses supplied by fertigation and of calcium by foliar spray on plant growth, fruit yield and quality of soilless grown strawberries, plants were supplied with nutrient solutions at $\mathrm{K}^{+}$concentrations of $9\left(T_{1}\right) ; 6\left(T_{2}\right.$, control) and $4.28 \mathrm{mmol} \mathrm{L}^{-1}\left(T_{3}\right)$ and with the control nutrient solution $\left(T_{2}\right)$ supplemented once a week by spraying $\mathrm{CaCl}_{2}$ on shoot at concentrations of $2.5\left(\mathrm{~T}_{4}\right)$ and $5 \mathrm{~g} \mathrm{~L}^{-1}$ $\left(T_{5}\right)$. Plant growth was lower in the higher potassium concentration $\left(T_{1}\right)$ and the highest LAI was recorded in the higher $\mathrm{CaCl}_{2}$ dose $\left(T_{5}\right)$. Fruit yield was higher in $T_{2}$ and $T_{3}$, the titratable acidity (TA) was higher in $T_{1}$, while total soluble solids (TSS) and the ratio TTS/TA did not differ among treatments. It was concluded that high potasium concentration in the nutrient solution reduces plant growth, fruit yield and quality and that calcium supplied on shoot by $\mathrm{CaCl}_{2}$ reduces fruit production.

Key words: Fragaria $\mathrm{x}$ ananassa, substrate, nutrient solution, cations.

\section{INTRODUÇÃo}

O cultivo sem solo do morangueiro é uma técnica empregada em várias regiões do Brasil, permitindo obter elevada produção e maior ergonometria no manejo da cultura (MORAES \& FURLANI, 1999). Entretanto, na região Sul do país, as frutas produzidas com o uso dessa técnica são de qualidade mais baixa em relação àquelas produzidas na região Sudeste do Brasil. Essa diferença tem sido atribuída, tanto ao efeito das condições ambientais, quanto da composição e do manejo da solução nutritiva (ANDRIOLO et al., 2009a).

Concentrações de solução nutritiva com valores de condutividade elétrica (CE) entre 1,4 e 1,8dS $\mathrm{m}^{-1}$ (PARANJPE et al., 2003) e de até 2,0dS $\mathrm{m}^{-1}$ (SAROOSHI \& CRESSWELL, 1994) são apontadas na literatura como aquelas a serem empregadas para obter qualidade e produtividade de frutas de morango. Embora a concentração elevada da solução nutritiva possa aumentar a qualidade das frutas, pode também diminuir a produção. Os resultados de ANDRIOLO et

\footnotetext{
IDepartamento de Fitotecnia, Universidade Federal de Santa Maria (UFSM), Santa Maria, RS, Brasil, 97105-900. E-mail: andriolo@smail.ufsm.brjeronimo@pq.cnpq.br. *Autor para correspondência.
} 
al. (2009a) mostraram que, no cultivo sem solo de morango utilizando areia lavada na região central do Rio Grande do Sul, a elevação da CE de $0,9 \mathrm{dS} \mathrm{m}^{-1}$ para $3,7 \mathrm{dS} \mathrm{m} \mathrm{m}^{-1}$ aumentou a acidez das frutas em $14 \%$ e o teor de sólidos solúveis em $11,5 \%$, mas a produção foi reduzida em $22 \%$, e o tamanho das frutas foi reduzido em 35\%. Uma alternativa para obter alta produtividade sem comprometer a qualidade das frutas nesse sistema de cultivo seria modificar a proporção entre os íons na solução nutritiva, sem alterar o somatório de cargas. Assim, a concentração da solução não seria modificada e o equilíbrio iônico poderia favorecer a absorção de alguns íons, especialmente $\mathrm{K}^{+}$e $\mathrm{Ca}^{++}$, os quais poderiam melhorar a qualidade das frutas.

Na fisiologia das plantas, o potássio desempenha importante função, agindo na regulação das reações de síntese, enquanto o cálcio influencia a estrutura e a resistência da parede celular (TAIZ \& ZIEGER, 2004). A absorção desses nutrientes não depende somente da sua disponibilidade em torno das raízes, mas também da sua concentração, porque há um limite para o somatório dos cátions que podem ser absorvidos simultaneamente pela planta (GREENWOOD \& STONE, 1998). Os nutrientes em concentração mais elevada tendem a reduzir ou inibir a absorção daqueles em concentração mais baixa.

As relações iônicas K:N e K:Ca têm sido sugeridas como critérios de manejo da solução nutritiva para diminuir a malformação (SHARMA \& SINGH, 2008) e melhorar a qualidade das frutas de morango (LIETEN, 2006). Na composição das soluções nutritivas que têm sido empregadas no cultivo sem solo do morangueiro em diversos países, são encontradas concentrações catiônicas que variam entre $2,2 \mathrm{mmol} \mathrm{L}^{-1}$ e $7,5 \mathrm{mmol} \mathrm{L}^{-1}$ para o $\mathrm{K}^{+}$e entre $1,1 \mathrm{mmol} \mathrm{L}^{-1} \mathrm{e} 3,8 \mathrm{mmol} \mathrm{L}^{-1}$ para a $\mathrm{Ca}^{++}$ (GIMÉNEZ et al., 2008). Para a cultura do morango, cultivar 'Elsanta', em dois ciclos produtivos consecutivos no outono e na primavera, LIETEN (2006) observou que, no primeiro ciclo, diferentes relações $\mathrm{K}^{+}: \mathrm{Ca}^{2+}: \mathrm{Mg}^{2+}$ não afetaram o número, o tamanho e a produção de frutas, enquanto no segundo ciclo as relações baixas em cálcio inferiores a $2 \mathrm{mmol} \mathrm{L}^{-1}$ reduziram o crescimento vegetativo e o número, o tamanho e a produção de frutas. No Brasil, não foram encontrados na literatura resultados de pesquisas indicando o efeito de diferentes níveis de potássio e cálcio na produção e qualidade de frutas de morango, sendo necessárias investigações que permitam ao produtor obter cultivos com alta produção e qualidade por meio do manejo adequado da solução nutritiva.

O objetivo deste trabalho foi determinar o crescimento da planta, a produção e a qualidade de frutas de morango em cultivo sem solo, com diferentes concentrações de potássio na solução nutritiva e de doses de cálcio em aplicação na parte aérea das plantas.

\section{MATERIAL E MÉTODOS}

O experimento foi conduzido no Departamento de Fitotecnia da Universidade Federal de Santa Maria, no interior de um abrigo de $200 \mathrm{~m}^{2}$, coberto com polietileno de baixa densidade de $150 \mu \mathrm{m}$ de espessura. As unidades experimentais foram formadas por telhas de fibrocimento, com 3,60m de comprimento, $1,10 \mathrm{~m}$ de largura, canais de $0,06 \mathrm{~m}$ de altura e $0,18 \mathrm{~m}$ de distância entre os canais. Cada telha foi apoiada sobre suportes a $0,80 \mathrm{~m}$ de altura do solo, com declividade de $1 \%$, e a superfície foi revestida com filme transparente de polietileno de baixa densidade com espessura de $100 \mu \mathrm{m}$. Os canais foram preenchidos com brita basáltica média, de tamanho de partículas entre 0,015m e $0,020 \mathrm{~m}$ e sobre esta foi colocada uma tela antiinseto coberta com uma camada de $0,15 \mathrm{~m}$ de areia média como substrato para a sustentação das plantas. As características físicas da areia foram determinadas no Laboratório de Física do Solo da UFSM, indicando granulometria entre $0,001 \mathrm{~m}$ e $0,003 \mathrm{~m}$, densidade aparente de $1.608,6 \mathrm{~g} \mathrm{dm}^{-3}$ e capacidade de retenção de água de $198,6 \mathrm{~mL} \mathrm{dm}^{-3}$. Um reservatório de fibra de vidro com capacidade para 500L foi instalado próximo à extremidade inferior das telhas, para armazenar a solução nutritiva, conforme descrito em ANDRIOLO (2007).

A solução nutritiva de referência foi a recomendada por FURLANI \& FERNANDES JÚNIOR (2004), modificada para atingir relações iônicas de $\mathrm{NO}_{3}^{-}$ $/ \mathrm{K}^{+}$de 1,4 e K $\mathrm{K}^{+} /\left(\mathrm{Ca}^{++}+\mathrm{Mg}^{++}\right)$de 1,1 e somatório iônico de $14,2 \mathrm{meq} \mathrm{L}^{-1}$. As plantas foram cultivadas com solução nutritiva contendo $9\left(\mathrm{~T}_{1}\right), 6\left(\mathrm{~T}_{2}\right.$, testemunha) e 4,28mmol L $\mathrm{m}^{-1}$ $\left(\mathrm{T}_{3}\right)$ de $\mathrm{K}^{+}$e com a solução testemunha $\left(\mathrm{T}_{2}\right)$ suplementada uma vez por semana por pulverizações foliares de cálcio nas concentrações de 2,5 $\left(\mathrm{T}_{4}\right)$ e $5 \mathrm{~g} \mathrm{~L}^{-1}$ de $\mathrm{CaCl}_{2}\left(\mathrm{~T}_{5}\right)$, totalizando cinco tratamentos. As aplicações de $\mathrm{CaCl}_{2}$ iniciaram no florescimento, sendo utilizado um volume de calda de $20,8 \mathrm{~mL}$ por planta. Foi empregado o delineamento experimental inteiramente casualizado, com quatro repetições, sendo cada parcela composta por uma unidade do dispositivo de cultura, com 48 plantas. Os micronutrientes foram fornecidos, em mg $L^{-1}$, nas concentrações de 0,03 de Mo; 0,42 de B; 0,06 de $\mathrm{Cu} ; 0,50$ de $\mathrm{Mn} ; 0,22$ de $\mathrm{Zn}$ e 1,0 de Fe. As concentrações dos macronutrientes foram de $\mathrm{NO}_{3}^{-}-$ $\mathrm{NH}_{4}^{+}-\mathrm{K}^{+}-\mathrm{H}_{2} \mathrm{PO}_{4}^{-}-\mathrm{Ca}^{++}-\mathrm{Mg}^{++}-\mathrm{SO}_{4}^{-}$, em mmol L-1 , e o somatórios iônicos, em meq $\mathrm{L}^{-1}$, foram de, respectivamente, 9,66-0,33-9-2-3-2-2 e 14,0 em T ; 9,990,33-6-2-6-2-2 e 14,2 em T ; e 8,45-1,56-4,25-2-5,1-3,153,15 e 13,9 em $\mathrm{T}_{3}$. A relação iônica $\mathrm{K}^{+} /\left(\mathrm{Ca}^{2+}+\mathrm{Mg}^{2+}\right)$ foi

Ciência Rural, v.40, n.2, fev, 2010. 
de 1,80 em $\mathrm{T}_{1}$, de 0,75 em $\mathrm{T}_{2}$ e de 0,52 em $\mathrm{T}_{3}$. As concentrações iônicas dos tratamentos $T_{1}, T_{2}$ e $T_{3}$ foram estabelecidas de forma a manter similar o somatório de íons. Em $\mathrm{T}_{4}$ e $\mathrm{T}_{5}$, as concentrações de $\mathrm{CaCl}_{2}$ foram estabelecidas com base no protocolo experimental de TOIVONEN \& STAN (2001).

Os fertilizantes empregados foram nitrato de potássio $\left(\mathrm{KNO}_{3}\right)$, nitrato de cálcio $\left[\mathrm{Ca}\left(\mathrm{NO}_{3}\right)_{2}\right]$ (calcinit), monofosfato de potássio $\left(\mathrm{KH}_{2} \mathrm{PO}_{4}\right)$ e sulfato de magnésio $\left(\mathrm{MgSO}_{4}\right)$. Em T3, a concentração total de nitrogênio foi atingida empregando nitrato de amônio $\left(\mathrm{NH}_{4} \mathrm{NO}_{3}\right)$. O cálculo das relações iônicas entre os macronutrientes e das quantidades de sais micronutrientes foi feito de acordo com a metodologia descrita por ANDRIOLO (1999).

A CE foi medida diariamente e corrigida quando os valores situaram-se acima ou abaixo de $10 \%$ do valor original, mediante adição de água ou de volumes complementares de solução nutritiva. $\mathrm{O}$ pH também foi medido diariamente e corrigido sempre que um desvio de 0,2 unidade foi observado, mediante adição de soluções $1 \mathrm{~N}$ de $\mathrm{H}_{3} \mathrm{PO}_{4}$ ou $\mathrm{KOH}$, com volumes estimados a partir de uma curva de titulação previamente ajustada no laboratório. Volumes complementares de solução nutritiva preparada de acordo com a composição original de cada tratamento foram adicionados sempre que o volume medido no reservatório foi igual ou inferior a 50\% do volume original. Para tal, uma relação linear foi previamente ajustada entre a altura da coluna líquida e o volume contido no reservatório. Nenhum descarte de solução nutritiva foi feito durante o período experimental. $\mathrm{O}$ valor médio do $\mathrm{pH}$ durante todo o experimento foi de 5,5. A condutividade elétrica foi de $1,14 \mathrm{dS} \mathrm{m}^{-1} \mathrm{em} \mathrm{T}_{1}$, de 1,09dS m-1 em $\mathrm{T}_{2}, \mathrm{~T}_{4}$ e $\mathrm{T}_{5}$ e de $1,06 \mathrm{dS} \mathrm{m} \mathrm{m}^{-1} \mathrm{em}_{3}$.

A frequência das fertirrigações foi estimada com base na capacidade de retenção de água do substrato e na transpiração potencial da cultura, de forma a fornecer diariamente volumes de água superiores àqueles transpirados, com um coeficiente de drenagem igual ou superior a 30\%. A transpiração potencial foi estimada considerando-se a radiação solar global incidente no topo da cobertura vegetal e a área foliar da cultura (HENNION \& VESCHAMBRE, 1997), com base em dados de literatura sobre determinações similares feitas em hortaliças cultivadas no mesmo local e ambiente (DALMAGO et al., 2006). Por meio dessa estimativa, foram programadas quatro fertirrigações diárias de 15 min às 9, 11, 13 e 16h30min, controladas por um programador horário.

O plantio foi realizado no dia 9 de abril de 2008, na densidade de 12 plantas $\mathrm{m}^{-2}$, e o experimento foi encerrado em 20 de novembro de 2008. As mudas foram produzidas em bandejas de poliestireno de 128 células, obtidas a partir de pontas de estolões da cultivar 'Arazá', de origem uruguaia. A data de início do florescimento das plantas em cada tratamento foi considerada atingida quando $50 \%$ das plantas de cada parcela apresentaram pelo menos uma inflorescência em antese. As colheitas iniciaram em 10 de junho e foram feitas duas vezes por semana, na fase de maturação completa, correspondente ao estádio fenológico 87 (MEIER et al., 1994). As frutas colhidas foram pesadas, contabilizadas e conduzidas ao Laboratório Pós-colheita para determinar a firmeza, com penetrômetro de ponteira de $10 \mathrm{~mm}$, acidez titulável e teor de sólidos solúveis totais por refratometria (AOAC, 1990). Em cada colheita, foi determinada a massa seca de uma amostra de $100 \mathrm{~g}$ de frutas de cada tratamento, por meio da secagem da amostra em estufa de circulação forçada de ar, na temperatura de $65^{\circ} \mathrm{C}$, até obtenção de massa constante entre duas determinações consecutivas. A proporção entre a massa fresca e a massa seca foi empregada para estimar a massa seca total de frutas em cada colheita.

Ao final do período experimental, foram coletadas quatro plantas de cada tratamento para determinação da massa seca dos órgãos vegetativos da planta. A área foliar específica (SLA, em $\mathrm{m}^{2} \mathrm{~g}^{-1}$ ) de cada planta coletada foi determinada por meio da massa seca de 30 discos de $10 \times 10^{-4} \mathrm{~m}^{-2}$ de diâmetro. Um coeficiente de proporcionalidade foi calculado entre a massa seca e a superfície dos discos, o qual foi empregado para estimar o índice de área foliar (IAF) da planta. Foi considerada como massa seca vegetativa de cada planta a soma da massa das folhas, dos pecíolos, das inflorescências, coroa e raízes. Os resultados foram submetidos à análise de variância, e as médias dos tratamentos foram comparadas pelo teste de Tukey, a 5\% de probabilidade de erro.

\section{RESULTADOS E DISCUSSÃO}

O menor crescimento das plantas foi obtido com o tratamento de maior concentração de potássio (Tabela 1), enquanto que os tratamentos com as duas concentrações inferiores desse elemento $\left(T_{2}\right.$ e $\left.T_{3}\right)$ e com as duas doses de cálcio aplicadas na parte aérea $\left(\mathrm{T}_{4} \mathrm{e} \mathrm{T}_{5}\right)$ não diferiram significativamente na massa seca total e na vegetativa. Na comparação de $T_{1}$ com a média desses quatro últimos tratamentos, a redução foi de $52 \%$ na massa seca vegetativa e de $48,5 \%$ na massa seca total. O IAF diferiu entre os cinco tratamentos com valor mais elevado em $\mathrm{T}_{5}$. O menor valor do IAF foi observado em $\mathrm{T}_{1}$, com uma redução de $85 \%$ em relação ao maior valor, observado em $\mathrm{T}_{5}$. $\mathrm{O}$ efeito dos 
Tabela 1 - Crescimento da planta do morangueiro, cultivar 'Arazá', em cultivo sem solo com diferentes doses de potássio e cálcio. Santa Maria, UFSM, 2008.

\begin{tabular}{|c|c|c|c|c|}
\hline \multirow{2}{*}{ Tratamentos } & ----------.- & seca (g p & 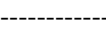 & \multirow{2}{*}{$\operatorname{IAF}\left(\mathrm{m}^{2} \mathrm{~m}^{-2}\right)$} \\
\hline & Vegetativa & Frutas & Total & \\
\hline $\mathrm{T} 1$ & $16,66 b^{*}$ & $17,90 \mathrm{~d}$ & $34,56 \mathrm{~b}$ & $0,51 \mathrm{e}$ \\
\hline $\mathrm{T} 2$ & $33,40 a$ & $40,14 a$ & $73,54 a$ & $2,69 \mathrm{c}$ \\
\hline T3 & $30,65 a$ & $37,60 \mathrm{a}$ & $68,25 a$ & $2,18 \mathrm{~d}$ \\
\hline $\mathrm{T} 4$ & $32,90 \mathrm{a}$ & $30,10 \mathrm{~b}$ & $63,00 \mathrm{a}$ & $3,10 \mathrm{~b}$ \\
\hline $\mathrm{T} 5$ & $42,06 a$ & $21,50 \mathrm{c}$ & $63,56 a$ & $3,41 \mathrm{a}$ \\
\hline $\mathrm{CV} \%$ & 13,78 & 9,23 & 8,79 & 10,32 \\
\hline
\end{tabular}

* Médias seguidas por letra minúscula distinta na coluna diferem entre si pelo teste de Tukey, a 5\% de probabilidade de erro. T1: 9mmol L $\mathrm{m}^{-1}$ de $\mathrm{K}^{+}$; T2: $6 \mathrm{mmol} \mathrm{L}^{-1}$ de $\mathrm{K}^{+}$; T3: 4,25mmol L ${ }^{-1}$ de $\mathrm{K}^{+}$; T4 e T5: $\mathrm{CaCl}_{2}$ semanal a 2,5g L ${ }^{-1}$ e $5 \mathrm{~g} \mathrm{~L}^{-1}$, respectivamente.

tratamentos sobre o crescimento das folhas foi mais intenso do que sobre o crescimento vegetativo das frutas.

A produção mais elevada de frutas foi obtida em $\mathrm{T}_{2}$ e $\mathrm{T}_{3}$, com massa fresca média de 551,85g planta $^{-1}$ (Tabela 2). Em ordem decrescente de produção de frutas, aparecem os tratamentos $\mathrm{T}_{4}, \mathrm{~T}_{5}$ e $\mathrm{T}_{1}$. Esses resultados indicam que, tanto as duas doses de cálcio, quanto a maior dose de potássio, reduziram a produção de frutas. Essa redução foi decorrência principalmente da diminuição no número de frutas, pois o tamanho não diferiu significativamente entre os tratamentos. É pouco provável que a emissão de inflorescências tenha sido afetada, porque essa é uma variável do desenvolvimento associada principalmente com a temperatura do ar e o fotoperíodo. A menor produção de frutas pode ser atribuída ao aborto de flores nas fases iniciais do seu desenvolvimento. Entretanto, as causas do aborto seriam diferentes no tratamento com a dose mais elevada de potássio $\left(\mathrm{T}_{1}\right)$ e naqueles relativos às doses de cálcio na parte aérea $\left(\mathrm{T}_{4} \mathrm{e} \mathrm{T}_{5}\right)$. Em $\mathrm{T}_{1}$, a causa seria o efeito combinado da baixa produção de assimilados, decorrente da redução no crescimento da área foliar (Tabela 1) e de perturbações na absorção de cálcio e/ou magnésio induzidas pelo potássio. Em $\mathrm{T}_{4}$ e $\mathrm{T}_{5}$, o crescimento vegetativo não foi reduzido, sugerindo que o cálcio afetou somente a produção de frutas, conforme observado por LIETEN (2006).

Com relação às variáveis da qualidade das frutas (Tabela 2), verificou-se que a firmeza não foi significativamente afetada pelos tratamentos, com média de 1,54N. A acidez foi mais elevada nas frutas do tratamento com a maior concentração de potássio $\left(\mathrm{T}_{1}\right)$, sendo $36,3 \%$ maior do que a média dos demais tratamentos, os quais não diferiram significativamente entre si. O teor de sólidos solúveis não diferiu significativamente entre os tratamentos, com média de $6,65^{\circ}$ Brix. A relação entre o teor de sólidos solúveis totais e a acidez total (SST/AT), empregada para indicar a qualidade organoléptica das frutas de morango, não diferiu entre os tratamentos, com média de $0,70^{\circ} \mathrm{Brix}$ (meq 100 $\left.\mathrm{mL}^{-1}\right)^{-1}$.

A principal hipótese que pode explicar a redução observada no crescimento e produção das

Tabela 2 - Produção, número, massa média das frutas, acidez titulável, teor de sólidos solúveis totais (SST) e relação entre o teor de sólidos solúveis totais e acidez titulável (SST/AT) de frutas de morango, cultivar 'Arazá', produzidas em cultivo sem solo com diferentes doses de potássio e cálcio. Santa Maria, UFSM, 2008.

\begin{tabular}{|c|c|c|c|c|c|c|}
\hline Tratamentos & $\begin{array}{l}\text { Produção } \\
\left.\text { (g planta }^{-1}\right)\end{array}$ & $\mathrm{N}^{\mathrm{o}}$ de frutas & $\begin{array}{l}\text { Massa média } \\
\left(\mathrm{g}_{\text {fruta }}{ }^{-1}\right)\end{array}$ & $\begin{array}{c}\text { Acidez } \\
\left.\text { (meq } 100 \mathrm{~mL}^{-1}\right)\end{array}$ & SST ( ${ }^{\circ}$ Brix) & $\begin{array}{c}\text { SST/AT } \\
{\left[{ }^{\circ} \text { Brix }\left(\mathrm{meq} 100 \mathrm{~mL}^{-1)}\right]^{-1}\right.}\end{array}$ \\
\hline $\mathrm{T}_{1}$ & $205,0 d^{*}$ & $23 \mathrm{c}$ & $8,9 a$ & $12,13 a^{*}$ & $7,33 \mathrm{a}$ & $0,60 \mathrm{a}$ \\
\hline $\mathrm{T}_{2}$ & 578,2 a & 55 a & $10,5 a$ & $9,10 \mathrm{~b}$ & $6,37 \mathrm{a}$ & $0,70 \mathrm{a}$ \\
\hline $\mathrm{T}_{3}$ & 525,5 a & 58 a & $9,1 \mathrm{a}$ & $9,50 \mathrm{~b}$ & $6,33 \mathrm{a}$ & 0,66 a \\
\hline $\mathrm{T}_{4}$ & $429,4 \mathrm{~b}$ & $41 \mathrm{~b}$ & $10,4 a$ & $9,00 \mathrm{~b}$ & $6,50 \mathrm{a}$ & $0,72 \mathrm{a}$ \\
\hline $\mathrm{T}_{5}$ & 338,7 c & $37 \mathrm{~b}$ & 9,1a & 8,03 b & 6,73 a & $0,84 \mathrm{a}$ \\
\hline CV\% & 5,55 & 4,65 & 10,5 & 10,14 & 12,57 & 14,45 \\
\hline
\end{tabular}

* Médias seguidas por letra minúscula distintas na coluna diferem entre si pelo teste de Tukey, a 5\% de probabilidade de erro.

$\mathrm{T}_{1}$ : 9mmol L $\mathrm{m}^{-1}$ de $\mathrm{K}^{+} ; \mathrm{T}_{2}$ : $6 \mathrm{mmol} \mathrm{L}^{-1}$ de $\mathrm{K}^{+} ; \mathrm{T}_{3}$ : 4,25mmol L $\mathrm{m}^{-1}$ de $\mathrm{K}^{+} ; \mathrm{T}_{4}$ e $\mathrm{T}_{5}$ : $\mathrm{CaCl}_{2}$ semanal a $2,5 \mathrm{~g} \mathrm{~L}^{-1}$ e $5 \mathrm{~g} \mathrm{~L}^{-1}$, respectivamente.

Ciência Rural, v.40, n.2, fev, 2010. 
plantas de morangueiro por efeito da dose mais elevada de potássio baseia-se na absorção competitiva em relação ao somatório total de cátions no interior da planta, como demonstrado por GREENWOOD \& STONE, (1998). Isso significa que a elevada concentração de potássio induziria deficiências de cálcio e magnésio, reduzindo o crescimento de toda a planta. Essa hipótese encontra confirmação nos resultados de LIETEN (2006), o qual observou redução no crescimento vegetativo, no número, no tamanho e na produção de frutas de morangueiro, no tratamento com a relação K:Ca:Mg mais elevada, que foi de 8:1,5:1.

A aplicação de cálcio na parte aérea das plantas reduziu a produção de frutas (Tabela 2). Esse efeito não pode ser atribuído a interações com o potássio, pois a solução nutritiva empregada nos tratamentos $\mathrm{T}_{4}$ e $\mathrm{T}_{5}$ foi a mesma de $\mathrm{T}_{2}$, o qual atingiu produção mais elevada. A tabela 2 indica que o efeito negativo do Ca ocorreu apenas na variável número de frutas por planta. Uma hipótese é que esse efeito seja consequência dos íons $\mathrm{Cl}^{-}$oriundos da dissociação do $\mathrm{CaCl}_{2}$. Em experimento anterior, ANDRIOLO et al. (2009b) observaram redução de crescimento e produção das plantas de morangueiro quando foi empregado o $\mathrm{KCl}$ em adubação pré-plantio, sugerindo efeito tóxico do cloro. Entretanto, ao empregar o sulfato de cálcio em aplicação foliar em plantas de morangueiro, DUNN \& ABLE (2006) observaram diminuição na qualidade das frutas, enquanto ESMEL et al. (2006) observaram redução na produção sem efeito na qualidade das frutas. Segundo esses autores, a distribuição irregular do cálcio na planta devido a sua baixa mobilidade seria uma das causas dessas observações contraditórias. Tanto os resultados atuais, quanto aqueles da literatura, colocam em dúvida a eficiência da fertilização foliar de cálcio no morangueiro, mesmo que sejam empregados fertilizantes solúveis sem cloro.

Embora a acidez das frutas tenha aumentado por efeito do potássio, a relação dessa variável com o teor de sólidos solúveis totais (SST/AT) foi inferior, significando frutos mais azedos ao paladar. Os resultados dessa pesquisa indicam que a elevação da concentração de potássio na solução nutritiva não é uma técnica que poderá ser empregada para aumentar a qualidade das frutas de morango no cultivo sem solo. A composição da solução nutritiva de $\mathrm{T}_{2}$ confirma-se como aquela a ser empregada nesse sistema de cultivo. A elevação da concentração de Ca na solução nutritiva sem alterar os demais íons é uma alternativa difícil de ser realizada, porque a fonte comercial disponível é o nitrato de cálcio e não é possível modificar apenas a concentração desse nutriente. Os resultados atuais sugerem ainda que devem ser tomadas precauções no manejo da adubação do morangueiro, no sistema convencional conduzido no solo coberto com filme de polietileno (mulching). Doses excessivas de adubação potássica poderão provocar consequências sobre o crescimento e a produção, semelhantes àquelas observadas nesse experimento.

\section{CONCLUSÕES}

O aumento da concentração de potássio na solução nutritiva diminui o crescimento, a produção e a qualidade organoléptica das frutas de morango. $\mathrm{O}$ fornecimento suplementar de cálcio na parte aérea das plantas por meio do $\mathrm{CaCl}_{2}$ reduz o número e a produção das frutas.

\section{AGRADECIMENTOS}

Ao Conselho Nacional de Desenvolvimento Científico e Tecnológico (CNPq), auxílio n. 470177/2006-3, pelas bolsas PQ a Jerônimo Luiz Andriolo, PIBIC à Francieli Lima Cardoso, BIC-CNPq a Odair José Schmitt, BIC-FAPERGS à Lígia Erpen e PIBITI a Djeimi Isabel Jänisch.

\section{REFERÊNCIAS}

ANDRIOLO, J.L. Fisiologia das culturas protegidas. Santa Maria: UFSM, 1999. 142p.

ANDRIOLO, J.L. et al. Concentração da solução nutritiva no crescimento da planta, na produção e na qualidade de frutas do morangueiro. Ciência Rural. v.39, n.3, p.684-690, 2009. Disponível em: <http://www.scielo.br/scielo.php?script=sci_arttext\&pid=S010384782009000300009\&lng=pt\&nrm=iso >. Acesso em: 15 mai. 2009. doi: 10.1590/S0103-84782009005000008.

ANDRIOLO, J.L. et al. Cultivo sem solo do morangueiro com três métodos de fertirrigação. Ciência Rural, v.39, n.3, p.691695, 2009. Disponível em: <http://www.scielo.br/ s cielo.ph p ? s c ri pt = sci_art text \& pid = S 0103 84782009000300010\&lng=pt\&nrm=iso $>$. Acesso em: 15 mai. 2009. doi: 10.1590/S0103-84782009005000009.

ANDRIOLO, J.L. Preparo e manejo da solução nutritiva na produção de mudas e de frutas do morangueiro. In: SEMINÁRIO SOBRE O CULTIVO HIDRÔPONICO DE MORANGUEIRO, 2007, Santa Maria, RS. Anais... Santa Maria: UFSM, CCR, Departamento de Fitotecnia, 2007. p.41-50.

ASSOCIATION OF OFFICIAL ANALYTICAL CHEMISTS. Official methods of analysis of the Association of Official Analytical Chemists. 15.ed. Arlington, 1990. 1298p.

DALMAGO, G.A. et al. Evapotranspiração máxima da cultura do pimentão em estufa plástica em função da radiação solar, temperatura, umidade do ar e déficit de saturação do ar. Revista Brasileira de Agrometeorologia, v.36, n.3, p.785-792, 2006. 
DUNN, J.L.; ABLE, A.J. Pre-harvest calcium effects on sensory quality and calcium mobility in strawberry fruit. Acta Horticulturae, v.708, p.307-312, 2006.

ESMEL, C.E. et al. Calcium thiosulfate does not influence yield, postharvest quality, or calcium content of 'Sweet Charlie' strawberry. Acta Horticulturae, v.708, p.97-102, 2006.

FURLANI, P.R.; FERNANDEZ JÚNIOR, F. Cultivo hidropônico de morango em ambiente protegido. In: CORRÊA ANTUNEZ, L.E. et al. (Ed.). SIMPÓSIO NACIONAL DO MORANGO \& ENCONTRO DE PEQUENAS FRUTAS E FRUTAS NATIVAS DO MERCOSUL, 2., 2004, Pelotas, RS. Anais... Pelotas: EMBRAPA, 2004. p.102-115. (Documentos 124).

GIMÉNEZ, G. et al. Cultivo sem solo no morangueiro. Ciência Rural, v.38, n.1, p. 273-279, 2008. Disponível em: <http:// www.scielo.br/scielo.php?script $=$ sci_arttext \&pid=S010384782008000100048\&lng=pt\&nrm=iso $>$. Acesso em: 18 mar. 2008. doi: 10.1590/S0103-84782008000100048.

GREENWOOD, D.J.; STONE, D. Prediction and measurement of the decline in the critical-K, the maximum-K and total cation plant concentration during growth of field vegetables crops. Annals of Botany, v.82 p.871-881, 1998.

HENNION, B.; VESCHAMBRE, D. La fraise: maîtrise de la production. Paris: CTIFL, 1997. 299p.

LIETEN, P. Effect of K:Ca:Mg ratio on performance of 'Elsanta' strawberries grown on peat. Acta Horticulturae, v.708, p.397-400, 2006.

MEIER, U. et al. Phänologische Entwick-lungsstadien des Kernobstes (Malus domestica Borkh. und Pyrus communis L.), des Steinobstes (Prunus-Arten), der Johannisbeere (Ribes-Arten) und der Erdbeere (Fragaria $x$ ananassa Duch.). Nachrichtenbl. Deutchland Pflanzenschutzd, v.46, p.141153, 1994.

MORAES, C.A.G. de; FURLANI, P.R. Cultivo de hortaliças de frutos em hidroponia em ambiente protegido. Informe Agropecuário, v.20, n.200/201, p.105-113, 1999 .

PARANJPE A. et al. Winter strawberry production in greenhouses using soilless substrates: an alternative to methyl bromide soil fumigation. Proceedings of the Florida State for Horticultural Science, v.116, p.98-105, 2003.

SAROOSHI, R.A.; CRESSWELL, G.C. Effects of hydroponic solution composition, electrical conductivity and plant spacing on yield and quality of strawberries. Australian Journal of Experimental Agriculture, v.34, p.529-535, 1994. Disponível em: <http://www.publish.csiro.au/?paper=EA9940529>. Acesso em 12 jan. 2008. doi:10.1071/EA9940529.

SHARMA, R.R.; SINGH, R. Fruit nutrient content and lipoxygenase activity in relation to the production of malformed and button berries in strawberry (Fragaria $x$ ananassa Duch.). Scientia Horticulturae, v.119, p.28-31, 2008. Disponível em: <http:// www.sciencedirect.com/science?_ob=ArticleURL\&_udi=B6TC34T5HJ5R>. Acesso em: 9 fev. 2009. doi: 10.1016/ j.physletb.2003.10.071

TAIZ, L; ZEIGER, E. Fisiologia vegetal. 3.ed. Porto Alegre: Artmed, 2004. 719p

TOIVONEN, P.M.A.; STAN, S. Effect of preharvest $\mathrm{CaCl}$ sprays on the postharvest quality of 'rainier' and 'totem ${ }^{3}$ strawberries. Acta Horticulturae. (ISHS) v.564, p.159163, 2001. Disponível em: <http://www.actahort.org/books/ 564/64_18.htm>. Acesso em 26 de fev. de 2008. 